Iberian Journal of the History of Economic Thought

ISSN-e 2386-5768

http://dx.doi.org/10.5209//JHE.58089

\title{
Evocación del historiador Vicent Llombart
}

Salvador Almenar Palau ${ }^{1}$

Received: 20/11/2017 / Accepted: 21/11/2017

Resumen. Esta evocación muestra cómo la formación inicial que recibió Vicent Llombart en el Seminario de historia del pensamiento económico en la Universidad de Valencia fue decisiva, pero no suficiente, para entender una trayectoria rotunda por sus contribuciones. Su propósito decidido, su firme exigencia personal y su apertura intelectual permanente nos ayudan a comprender mejor a un gran historiador.

Palabras clave: Vicent Llombart; biografía; Universidad de Valencia.

\section{[en] Remembrance of the historian Vicent Llombart}

\begin{abstract}
This remembrance shows how the initial training that Vicent Llombart received at the Seminar on the History of Economic Thought at the University of Valencia was crucial, although not the only element, to understanding a prominent academic career with so relevant contributions. His determined purpose, his self-demanding character and his permanent intellectual openness help us to better understand a great historian.
\end{abstract}

Keywords: Vicent Llombart; biography; University of Valencia.

JEL Classification: B32

La ausencia fatal de un buen amigo, a la vez colega y compañero durante décadas, aviva la memoria y convida a la melancolía. En estos párrafos me limitaré a evocar, entre tantos recuerdos, los primeros años de formación como historiadores del pensamiento económico y a esbozar una guía esencial sobre su intenso y fértil itinerario como historiador.

La dedicación vocacional y profesional de Vicent Llombart al estudio y la enseñanza de la Historia del pensamiento económico se debe, en gran medida, a la impronta intelectual de Ernest Lluch. Fue alumno suyo durante el curso 1970-1971 en dos asignaturas, y en el curso siguiente se incorporó al grupo inicial de colaboradores de Lluch dentro del Seminario de Historia de las doctrinas económicas - Santiago Lanzuela, Segundo Bru y el que escribe estas líneas-, todos recién licenciados de la primera promoción de economistas de la Universidad de Valencia.
En el Seminario participamos en diversas actividades formativas, empezando por las más sencillas, como la elaboración en equipo de varios ficheros bibliográficos de los economistas españoles y de la historiografía disponible sobre los mismos, la cuantificación de la literatura económica internacional de los siglos XVI al XIX, la presentación ante el grupo de clases simuladas, el seguimiento de los cursos de doctorado y la redacción de las primeras recensiones de libros recientes - nuestra prueba de fuego conjunta fueron dos volúmenes de textos de Quesnay-.

Conviene recordar que durante esos años se manifestó en las principales revistas internacionales una especial atención por algunos economistas del pasado, de Quesnay a Keynes, mientras se consolidaba la nueva History of Political Economy y proliferaban reuniones regulares especializadas en Gran Bretaña e Italia. Aunque en España el interés editorial se despertaba lentamente, también aparecie-

\footnotetext{
Universitat de València

Salvador.Almenar@uv.es

https://orcid.org/0000-0002-8493-2555
} 
ron algunas publicaciones de relieve sobre la historia del pensamiento económico - cuatro ejemplos: el libro de Pedro Schwartz sobre Stuart Mill, la Historia de Schumpeter, el volumen compilado por Spengler y Allen, o los Ensayos de Fabián Estapé-. La materia, a pesar de carecer de una tradición académica perfectamente definida $-\mathrm{O}$, en parte, justamente por ello_-, parecía prometer una atalaya muy atractiva para los jóvenes investigadores; una atalaya entonces agitada por la discusión sobre los paradigmas y las revoluciones científicas, pero también por los debates en la Historia económica - que seguíamos con la ayuda de Mario García Bonafé-.

Muy pronto Lluch nos planteó la necesidad de afrontar la elaboración de un proyecto de tesis doctoral, sujeta a la restricción de que implicara algunas aportaciones originales sustantivas. Tal restricción parecería obvia en la actualidad pero no era la norma seguida con frecuencia en las facultades de economía españolas de la época, donde abundaban los casos de tesis que, caritativamente, cabía definir como estados de la cuestión sobre un tema. Frente a este peligro habitual, los "refritos", cabía perseguir virtudes académicas como el rigor en el estudio de todas la fuentes primarias (exhaustividad) y secundarias (honesto reconocimiento), la profundidad frente a la simplificación, y una vigilante atención ante los cantos de sirena como el precursorismo, la tentadora tendencia a "descubrir mediterráneos" o las recetas mecánicas para interpretar las posibles relaciones entre el desarrollo de las ideas económicas y el ámbito histórico más general.

En reuniones formales o en conversaciones relajadas, a través de sugerencias de lecturas, notas y guiños, se fue perfilando una especie de heurística doméstica de la tesis doctoral y de la investigación en general. Con la ayuda de algunos autores que servían de ejemplo o de brújula y de otros utilizados como cartógrafos auxiliares: Cannan, Robbins, Schumpeter, Sraffa, Stigler, Viner; pero también Blaug, Meek, Spengler, Sowell, Hutchison, Coats, Collison Black, Winch, Gerschenkron, Napoleoni, Barucci, Garegnani, Macchioro o Venturi, entre otros referentes iniciales.

Entre los posibles temas de tesis doctoral se encontraban algunos periodos o autores españoles todavía no (bien) estudiados por una historiografía dispersa e irregular, a pesar de ciertas excepciones. De hecho, la secuencia historiográfica desde Estapé a Lluch era sin- gular en el mundo académico español. La tesis doctoral que Lluch había dedicado al pensamiento económico en Cataluña desde 1760 a 1840 abrigaba una defensa de homologación disciplinar. Postulaba la necesidad de estudiar, en primer término, los procesos de difusión, adaptación y reformulación de las ideas económicas acaecidos en los países "receptores" con el fin de afrontar el análisis y la transformación de unas situaciones de desarrollo cultural, institucional y económico diferentes a las de los países "emisores". Además, el estudio de estas transformaciones del conocimiento económico era imprescindible para evaluar la proyección histórica de las diferentes - o coincidentespercepciones, análisis y políticas económicas. En tercer lugar, el nuevo enfoque "nacional" del pensamiento económico no pretendía ser una alternativa a la historia del análisis económico sino una ampliación del ámbito de estudio con el que complementar la comprensión del papel desempeñado por las ideas económicas con una perspectiva histórica más amplia.

El primer trabajo de Vicent Llombart, sobre la difusión del Essay de Malthus en España (1973), se inscribe en un proyecto tentativo de tesis sobre la "introducción de la Escuela Clásica" en España y se publicó como un homenaje al desaparecido historiador Robert Sidney Smith, cuyos estudios representaban una avanzada del nuevo enfoque. Llombart presentaba las ideas de Malthus, aportaba testimonios adicionales que ampliaban el alcance de su difusión durante la primera mitad del ochocientos y apuntaba una hipótesis sobre la paradójica coincidencia entre la extendida mentalidad poblacionista y la difusión maltusiana.

Durante ese mismo año 1973 decidió dedicar su tesis al pensamiento económico del conde de Campomanes, alentado por Ernest Lluch, por la reapertura del archivo personal donde se encontraban numerosos textos inéditos $y$, en definitiva, por la relevancia histórica del personaje. El trabajo recopilatorio y el estudio crítico de los textos contribuyó decisivamente a la elaboración de una nueva y ordenada secuencia de las formulaciones de Campomanes que sustentaban un valioso cuadro de conjunto alternativo al que mostraba una ya extensa historiografía previa. No hace falta insistir, pero tampoco subestimar, en las limitaciones materiales de la investigación en aquella era pre-digital, o la extenuante combinación de docencia anual en dedicación exclusiva con la 
redacción de la tesis, todo ello combinado con las actividades cívicas y políticas.

Vicent presentó una síntesis inicial de los resultados sobre Campomanes en las primeras jornadas de Historia del pensamiento económico (Madrid, febrero de 1974), que reunió a los nuevos investigadores - casi todos economistas de Madrid, Barcelona y Valencia-, donde actuó también como secretario. La percepción de que una parte de sus interpretaciones acerca de Campomanes apareciera reflejada en una publicación ajena posterior reforzó su determinación y trabajo, pero también su preferencia por la comunicación científica escrita.

Leímos la tesis doctoral en julio de 1976 ante el mismo tribunal - los doctores Tomás y Valiente, Schwartz, Fontana y Lluch, presididos por el decano Sánchez Ayuso en substitución del doctor Fuentes Quintana-. Segundo Bru había defendido la suya sobre Sismondi unos días antes. La tesis de Llombart alberga una novedosa interpretación de la trayectoria intelectual de Campomanes como exponente de la "Ilustración oficial". En particular quiero destacar su juicio sobre la relevante proximidad inicial con el mercantilismo tardío y su evolución hacia un "agrarismo mercantilista" caracterizado por un deseable impulso demográfico en las zonas rurales con una política agraria que concordaba con la industria popular dispersa y aneja a la agricultura, así como una crítica coherente de los gremios y los consulados de comercio. Inmediatamente la tesis se proyectó en diferentes artículos y comunicaciones.

Una trayectoria más que prometedora quedó, sin embargo, comprometida por diferentes vicisitudes que dificultaron su estabilidad como docente en la Universidad de Valencia al final de los años setenta. En 1979 Llombart inició un nuevo itinerario. Primero en la Universidad Complutense durante cuatro cursos como profesor de Historia de las doctrinas económica junto a Pedro Schwartz, donde consolidó unas relaciones intelectuales y profesionales trascendentes. Prosiguió después con su colaboración destacada en la administración preautonómica valenciana y concluyó como Conseller de Obras Públicas entre 1983 y 1985.

\section{Propósito y culminación}

Al retornar a la universidad valenciana en calidad de profesor titular, a mi juicio Vicent comenzó no sólo un nuevo ciclo profesional sino personal o vital, en el amplio sentido de la expresión. El reencuentro con la vida académica comenzó al afrontar de inmediato las actividades docentes - en una Facultad desbordada por el número de estudiantes- y la recuperación de la investigación sobre los problemas abordados en la tesis. Más que un regreso mecánico, Vicent emprendió una verdadera reconstrucción.

La primera aportación más notable de ese arranque es la introducción y edición en 1988 de las Reflexiones sobre el comercio español a Indias (1762), un manuscrito en el que Campomanes manifestaba, según Llombart, su cercanía intelectual con el mercantilismo tardío, establecía una renovada interpretación crítica de la decadencia económica española y, como colofón, proponía la organización y extensión del comercio colonial español que exigía, entre otras medidas, la abolición del monopolio del puerto gaditano. El esfuerzo más apreciable se muestra, sin embargo, en el amplio abanico de trabajos suyos que aportaban un replanteamiento de las políticas agrarias ilustradas, sobre el comercio de granos y, en definitiva, sobre la significación intelectual e histórica de las distintas propuestas de reforma económica de la Ilustración española.

El resultado más denso de ese proceso de trabajo incesante es el libro de 1992 Campomanes, economista y político de Carlos III. El autor deja constancia de que se trata de un "producto notablemente diferente de aquella [tesis doctoral] en su contenido, en su estructura, en su objetivos y en sus conclusiones. Es un libro escrito línea a línea de nuevo, con investigación documental adicional...". Pausado y sobrio, el volumen presenta primero la evolución general del personaje, un destacadísimo ilustrado versado en ámbitos intelectuales y políticos aparentemente dispares - vistos con una óptica disciplinar actual- aunque entrelazados por un proyecto coherente y pragmático en su tiempo. Este análisis le permite presentar en detalle el pensamiento económico de Campomanes en las partes segunda y tercera, donde emergen aportaciones de relieve sobre las raíces y la originalidad, los intentos y resultados, ahora ya considerados más ampliamente sin la rigidez de los moldes o etiquetas tradicionales sobre el mercantilismo o el liberalismo, fruto de una revisión historiográfica más profunda. El epílogo "Ilustración, economía y poder" fluye como una reflexión detallada 
y madura sobre el papel y la influencia de las ideas, pero también de la actividad política, de Campomanes no en un decurso histórico abstracto sino en el de la monarquía española de la época. La ingeniosa propuesta de entender la visión de Campomanes, en parte, con el lema de "más mercado y más mercado" es solo una de sus respuestas laterales a las interpretaciones emergentes sobre el personaje y el periodo. Ernest Lluch en el prólogo al libro insinuaba, diplomáticamente, algunas sugerencias pero concluía sin reservas que "uno tiene la misma sensación de aquel campesino que retiró a su hijo de la escuela puesto que pensaba que el maestro ya no tenía nada más que enseñarle. En este caso sí es cierto".

El libro contiene numerosas alusiones sobre Aranda, Cabarrús, Ensenada, Floridablanca, Jovellanos, Olavide o Ward, pero no por casualidad. Porque, en paralelo a la restitución histórica de Campomanes, Llombart había desplegado un ambicioso proyecto de estudio sobre la economía política y la política económica de la Ilustración española, beneficiándose también de las aportaciones provenientes de la historia económica, y con la colaboración de un amplio grupo de colegas. A mi juicio destacan tres líneas específicas de investigación que han merecido la atención de muchos investigadores posteriores ante la solidez de sus resultados.

La primera se refiere a la difusión e influencia de los economistas europeos en España, que ya había abordado con Malthus, pero ahora dedicada al periodo de la Ilustración. Uno de los problemas del programa enunciado por Lluch sobre la historia "nacional" del pensamiento económico dejaba abierta o implícita una propuesta formal sobre las razones de la transferencia transnacional de las ideas económicas. Llombart intentó avanzar en ese campo en 1993 con una adaptación del modelo del mercado de las ideas propuesto por George Stigler, que reelaboró y defendió su aplicación para interpretar la recepción de la fisiocracia en España. El éxito de su artículo de 1995 en la revista EJHET fue inmediato y duradero, aunque no fue su última palabra. Sistematizó después una relación de las traducciones españolas de economía entre 1700 y 1812 . Y propuso una redefinición del esquema original, entendido como un proceso de circulación internacional del conocimiento económico, más cercano al modelo general de comunicación condicionado por factores históricos internos y externos.
La segunda línea específica sobre Jovellanos, en colaboración con otros colegas asturianos pero en especial con Joaquín Ocampo, se centró en el Informe de la Ley Agraria pero desembocó en el 2000 en un estudio complejo de sus obras económicas y, finalmente en 2012, en un volumen fascinante de interpretación y síntesis general del personaje, Jovellanos y el siglo de las luces, repleto de nuevas perspectivas y conclusiones expuestas a través de una narración simultáneamente analítica y diacrónica. En suma, uno de los mejores ejemplos de equilibrio entre erudición, profundidad de análisis y claridad expositiva que rebasaba necesariamente el ámbito de la economía, para abarcar una perspectiva histórica general.

La tercera línea es uno de los logros más apreciados de los lectores de Llombart: su síntesis del pensamiento económico de la Ilustración española, contenido en el estudio introductorio preparado para el tercer volumen de Economía y Economistas españoles, dirigida por el profesor Fuentes Quintana. Es un trabajo que trasciende la tarea de ordenar o cartografiar un universo plagado de estrellas y planetas con incontables relaciones mutuas, y se convierte en la disección de un proceso histórico sujeto a específicas condiciones de continuidad y cambio, acuerdos, disputas y consecuencias.

$$
* * *
$$

La secuencia de aportaciones de Vicent Llombart reseñadas hasta aquí es sumaria e incompleta. Injusta también por no insistir en ciertas virtudes de su estilo, su minuciosa preocupación por los conceptos y los títulos, y sobre todo por la medida y proverbial ironía, pura mayéutica. Invito al lector a que repase el estudio sistemático del profesor Pablo Cervera. Mi propósito era mostrar cómo la formación inicial que recibimos en el Seminario de historia del pensamiento económico en la Universidad de Valencia fue decisiva, pero no suficiente, para entender una trayectoria rotunda por sus contribuciones. Su propósito decidido, su firme exigencia personal y su apertura intelectual permanente nos ayudan a comprender mejor a un gran historiador.

Concluyo celebrando la iniciativa del Consejo de la revista Iberian Journal of the History of Economic Thought en dedicar un espacio singular para recordar a Vicent Llombart, que tanto empeño tuvo en su nacimiento y desarrollo. 


\section{Referencias}

Almenar, S. y Llombart, V. (1973) “Anotaciones a la introducción del 'Ensayo sobre la población', de Malthus en España”. Moneda y Crédito (126), septiembre, 79-86.

Llombart, V. (1976a) "Pensamiento y teoría de la política económica del conde de Campomanes. Economía e ideología de la 'Ilustración oficial' en España (1760-1790)". Tesis doctoral. Valencia: Universidad de Valencia, Facultad de Ciencias Económicas y Empresariales. Dirigida por E. Lluch.

- (1988a) Reflexiones sobre el comercio español a Indias. 1763. Edición, estudio preliminar y transcripción de Vicent Llombart. Madrid: Instituto de Estudios Fiscales y Ministerio de Hacienda, Clásicos del Pensamiento Económico Español. Reseña en (1989): Revista de Historia Económica (1), año VII, invierno, 223-227.

- (1992) Campomanes, economista y político de Carlos III. Madrid: Alianza.

- (1993) "Utility, market for public ideas and reception of Physiocracy: suggestions from an Spanish perspective". Ponencia. En "La diffusion internationale de la Physiocratie", Paris, ENS Fontenay-St. Cloud, Centre Alexandre Vandermonde pour l'Histoire de la Pensée Économique, 23-24 de septiembre. Provisional Draft in May, 19 pp. in folio. Existe separata.

- (1994a) "El mercado de ideas de Stigler y la transmisión internacional de los conocimientos económicos: una aplicación al caso español”. Ponencia. Valencia: Universitat de València, Facultat de Ciències Econòmiques i Empresarials, Seminario de Historia Económica del Departamento de Análisis Económico, 13 de mayo.

- (1994b) "El mercado de ideas de Stigler y la transmisión internacional de los conocimientos económicos: una aplicación al caso español". Ponencia. Madrid: Universidad Complutense de Madrid, Instituto Universitario de Economía de Mercado, Facultad de Ciencias Económicas y Empresariales, Departamento de Historia e Instituciones I. 18 de mayo.

- (1995a) "Mercado de ideas y recepción de la Fisiocracia en España: algunas sugerencias analíticas e históricas". Documento de trabajo. Valencia: Facultat de Ciències Econòmiques i Empresarials de la Universitat de València, Quaderns de Treball (3).

- (1995b) "Market of ideas and reception of Physiocracy in Spain: some analytical and historical suggestions". The European Journal of the History of Economic Thought (2:1), spring, 29-55.

- (2000a) Gaspar Melchor de Jovellanos. Escritos económicos. Estudio introductorio de Vicent Llombart. Madrid: Real Academia de Ciencias Morales y Políticas, Ministerio de Economía y Hacienda, Instituto de Estudios Fiscales, Fundación ICO, Clásicos del Pensamiento Económico Español, nueva época. Prólogo de Enrique Fuentes Quintana.

- (2000b) "Campomanes, el economista de Carlos III". En Fuentes Quintana, E. (Ed.): Economía y economistas españoles. Madrid: Galaxia Gütenberg-Círculo de Lectores, vol. III, 201-255.

- (2000c) "El Informe sobre la Ley Agraria de Jovellanos: núcleo analítico, programa de reformas y fuentes intelectuales". En Fuentes Quintana, E. (Ed.): Economía y economistas españoles. Madrid: Galaxia Gütenberg-Círculo de Lectores, vol. III, 421-445.

- (2013) Jovellanos y el otoño de las Luces. Educación, economía, política y felicidad. Gijón: Trea. 